\title{
Mathematikausbildung - die Diskussion braucht mehr Perspektiven!
}

\author{
Brigitte Lutz-Westphal
}

\begin{abstract}
In den letzten Monaten ist die Diskussion um die Mathematikausbildung in Schulen und Hochschulen wieder einmal aufgeflammt. Wir möchten sie in den „Mitteilungen der DMV“ nun versachlichen und durch einen verstärkten Blick in die Unterrichtspraxis und die Beschäftigung mit Forschungsergebnissen aus der Mathematikdidaktik besser fundieren. Ein multiperspektivischer Blick auf das komplexe Thema der Mathematikausbildung und -vermittlung wird helfen, Schuldzuweisungen hinter sich zu lassen und einen konstruktiven Diskurs in Gang zu setzen.
\end{abstract}

Zu Beginn des Frühjahrs 2017 kam die Mathematikausbildung in Deutschland wieder einmal in das öffentliche Bewusstsein. Ein „Brandbrief“ prangerte Missstände in der schulischen Mathematikausbildung an, die zu Schwierigkeiten in der Ausbildung an Universitäten und Fachhochschulen führen. Dieser Brandbrief, aus dem Bereich der Fachhochschulen kommend und überwiegend von Lehrenden an Universitäten und Fachhochschulen unterschrieben, führte zu Stellungnahmen von Seiten der Mathematikdidaktik, von der Mathematik-Kommission Übergang Schule-Hochschule der DMV, GDM und MNU und anderen $([1,2,3])$. Auch wenn hier durchaus unterschiedliche Positionen vertreten sind, etwa bezogen auf die Benennung von Ursachen oder bezogen auf die Entwicklungstrends, so stimmen doch alle Unterzeichnenden überein, dass die schulische und hochschulische Mathematikausbildung in Deutschland derzeit nicht optimal gelingt, und dass es dringend nötig ist, Veränderungen herbeizuführen.

In dieser und kommenden Ausgaben der Mitteilungen soll dieses Thema weiterhin in den Blick genommen werden, besonnen und ohne Schuldzuweisungen. Die Idee dabei ist folgende: Wir ermöglichen Einblicke in schulischen Mathematikunterricht und mathematische Hochschullehre sowie in fachdidaktische Überlegungen und Forschungsergebnisse zu diesem Themenkreis und nehmen dabei möglichst viele unterschiedliche Perspektiven ein. Damit schaffen wir eine solide Basis für eine fruchtbare und vor allem konstruktive Diskussion. Vermutlich brauchen wir noch mehr Annäherung und viel mehr gegenseitiges Zuhören als bisher, um durchschlagende Lösungsansätze entwickeln zu können. Ein verbesserter Dialog mit der Politik ist ein längerfristiges Ziel, das sich viel besser erreichen lässt, wenn wir erst einmal untereinander in einen engeren Austausch treten.

Die Mathematikausbildung an Schulen und Hochschulen ist eine komplexe Angelegenheit. Daher sollte man sich mit einer Vielzahl an Fragestellungen beschäftigen.
Das könnten in diesem Rahmen zunächst folgende sein:

- Was liegt im Unterrichts- und Lehralltag im Argen? Wie sind die Rahmenbedingungen?

- Was läuft gut (Best Practice)?

- Was würden wir uns wünschen?

- Problemthema Bruchrechnung

- Problemthema Stochastik

- Was fehlt den Studienanfängerinnen und -anfängern? Was können Abiturientinnen und Abiturienten überhaupt können?

- Was wird von den Studienanfängerinnen und -anfängern verlangt? Gibt es eine Passung zu den Abituranforderungen?

- Welche Problemstellen gibt es in der Lehrkräfteausbildung in allen Phasen?

- Wie funktioniert die Kommunikation mit der Politik? Was wäre da wünschenswert?

Weitere Fragestellungen werden sich aus der Diskussion ergeben, die hier durch drei Leitaspekte gegliedert wird: Diskussion - Unterrichtsrealität - Fachdidaktik.

\section{Diskussion}

Es wird Raum für fachlich fundierte Überlegungen zu den bereits geleisteten Diskussionsbeiträgen geben.

\section{Unterrichtsrealität}

Wir werden einen intensiven Blick in die Unterrichtspraxis werfen und uns ein Bild davon machen, wie Mathematikunterricht in der Realität aussieht. Hierzu gehören authentische Einblicke in den Unterrichtsalltag von Mathematiklehrkräften sowie Best-Practice-Beispiele und Projekte zur Unterrichtsentwicklung.

\section{Fachdidaktik}

Ein dritter Aspekt soll die Auseinandersetzung mit fachdidaktischen Fragen sein: Welche Forschungsergebnisse gibt es beispielsweise zum Übergang Schule-Hochschule? Was wissen wir im Rahmen der Mathematikdidaktik über 
das offenkundig vorhandene Problem mit der Bruchrechung? Hierzu sollen informative Fachbeiträge erscheinen, die den an der Diskussion Interessierten die Möglichkeit geben, sich ein breiteres Bild der Sachlage zu machen.

Ein wichtiger Beitrag im Rahmen der so gegliederten Diskussion sollen „O-Töne“ von Lehrkräften, Referendarinnen und Referendaren sowie von Schülerinnen und Schülern sein, die hier anonym veröffentlich werden, um authentische Aussagen zu bekommen. Eine erste Erfahrung hierzu ist, dass auf die Anfrage, die ausschließlich an uns persönlich bekannte Personen gerichtet war, nur sehr wenige Beiträge als Rücklauf kamen. Ist hier vielleicht ein erstes interessantes Indiz zu entdecken? Haben die Lehrkräfte einfach „nur“ keine Zeit oder ist aus Sicht der Schulpraxis von solch einer in Wissenschaftskreisen geführten Diskussion nichts Brauchbares zu erwarten? „Lohnt“ es sich nicht, Zeit für ein offenbar erfahrungsgemäß aussichtsloses Unterfangen einzusetzen? Es stellt sich also die Frage, wie wir an dieser Stelle besser ins Gespräch und den so wichtigen Austausch kommen können.

Umgekehrt sollten die Lehrenden an den Universitäten und Fachhochschulen es sich nicht nehmen lassen, sehr genau auf die Praxiserfahrungen der Lehrkräfte und Lernenden an den Schulen zu hören - abseits von empirischen Befunden, deren Ziel verallgemeinerte Aussagen sind, die genau nicht die individuellen Erfahrungen und Problematiken im Unterricht darstellen können. Diese jeweils individuellen Sichtweisen und Bewältigungsstra- tegien sind genau das, was bei den Schülerinnen und Schülern direkt ankommt. Daher soll einem ungeschönten individuellen Blick auf die Situation in den MathematikKlassenzimmern hier ein relativ breiter Raum gegeben werden.

Auch von Seiten der Wissenschaft ist eine gewisse Zurückhaltung zu bemerken. Nicht alle angefragten Autorinnen und Autoren können es sich vorstellen, in diesem Rahmen etwas beizutragen. Der Tonfall dieser Diskussion war bisher oft eher scharf, es drohen eventuell unschöne Reaktionen. Auch hieran wollen wir arbeiten und eine einladende Diskussion gestalten, die auch für leisere Töne offen ist, und für die es sich wirklich lohnt, seinen eigenen Beitrag zu leisten.

Bitte zögern Sie nicht, zu den beschriebenen Aspekten Diskussion, Unterrichtsrealität und Fachdidaktik Beiträge einzusenden. Wir hoffen auf eine anregende, faire und sachliche Annäherung an das komplexe Thema „Mathematikausbildung"!

[1] https://bildung-wissen.eu/fachbeitraege/schule-und-unterricht/ mathematik-brandbrief-gegen-bildungsstandards.html (22.9.2017)

[2] www.tagesspiegel.de/downloads/19590132/1/ mathematiker-distanzieren-sich-vom-mathematiker-brandbrief. pdf (22.9. 2017)

[3] Zur aktuellen Diskussion über die Qualität des Mathematikunterrichts - Stellungnahme von DMV, GDM und MNU, Mitteilungen der DMV 25-1 (2017), S. 6-7.

\title{
Mathematikunterricht - so sieht die Realität aus
}

\author{
Eine Mathematik-Referendarin an einem Hamburger Gymnasium berichtet
}

Ich unterrichte derzeit eine gutbehütete sechste Klasse an einem Gymnasium in Hamburg. Themen wie Bruchrechnung, Einführung in die Stochastik und ganze Zahlen stehen, beziehungsweise standen in diesem Schuljahr auf dem Lehrplan. Ja, alle diese „Hammerthemen“ darf ich meinen Schülerinnen und Schülern in einem Schuljahr beibringen. Ein Glück, dass ich die Klasse bereits aus einem Lehrauftrag kenne und dadurch die zwei bis drei Wochen Kennenlernen zu Beginn des Schuljahres wegfallen. Einige meiner Referendarskolleginnen und -kollegen haben es da nicht so gut getroffen, da sie direkt aus der Uni kamen, eine Woche vor Beginn des Schuljahres erfahren haben, welche Klassen sie unterrichten werden und nun erst einmal Rahmenpläne, Curricula, etc. durchforsten müssen, um überhaupt herauszubekommen, was wer wie und bis wann können muss. Das ist aber eine andere Geschichte ...

Ich habe mich entschlossen, zunächst einmal die ganzen Zahlen zu behandeln - ganz im Sinne der Zahlbereichserweiterung. Das ist mir, das möchte ich nun einmal behaupten, auch ganz gut gelungen. Die Schülerinnen und Schüler haben verstanden, wozu man diese neuen Zahlen braucht und auch wie man mit ihnen umgeht. Wir haben Geschichten zu dem neuen Thema geschrieben und auch viel gerechnet; alles gut. Auch die plötzlich anstehende Klassenarbeit fiel sehr gut aus, beinah ein bisschen zu gut.

Bei unserem nächsten Thema, der Bruchrechnung, waren die Kinder zunächst total begeistert - sie fanden Brüche toll! - und wir haben viel Zeit damit verbracht, die verschiedenen Bruchvorstellungen intensiv zu üben und anzuwenden. Doch plötzlich musste schon wieder eine Klassenarbeit geschrieben werden, die Termine werden durch die Schule vorgegeben. Also mussten Hals über Kopf - ohne eine tolle und ausführliche Einführung, wie sie die Schülerinnen und Schüler eigentlich benötigt hätten - nun die Rechenregeln eingeführt werden. Schade eigentlich, denn dadurch ist sehr viel an Vorbereitungszeit zunichtegemacht worden. Wie erwartet fiel diese Klassenarbeit nicht so gut aus wie die vorherige, was bedeutete, dass die Klassenarbeit wiederholt werden musste. Wäre es da nicht einfacher gewesen, die Klassenarbeit von vornherein später zu schreiben? Gerne hätte ich den Schülerinnen und Schülern den Unterricht in der Intensität angeboten, den sie benötigen, doch das war 


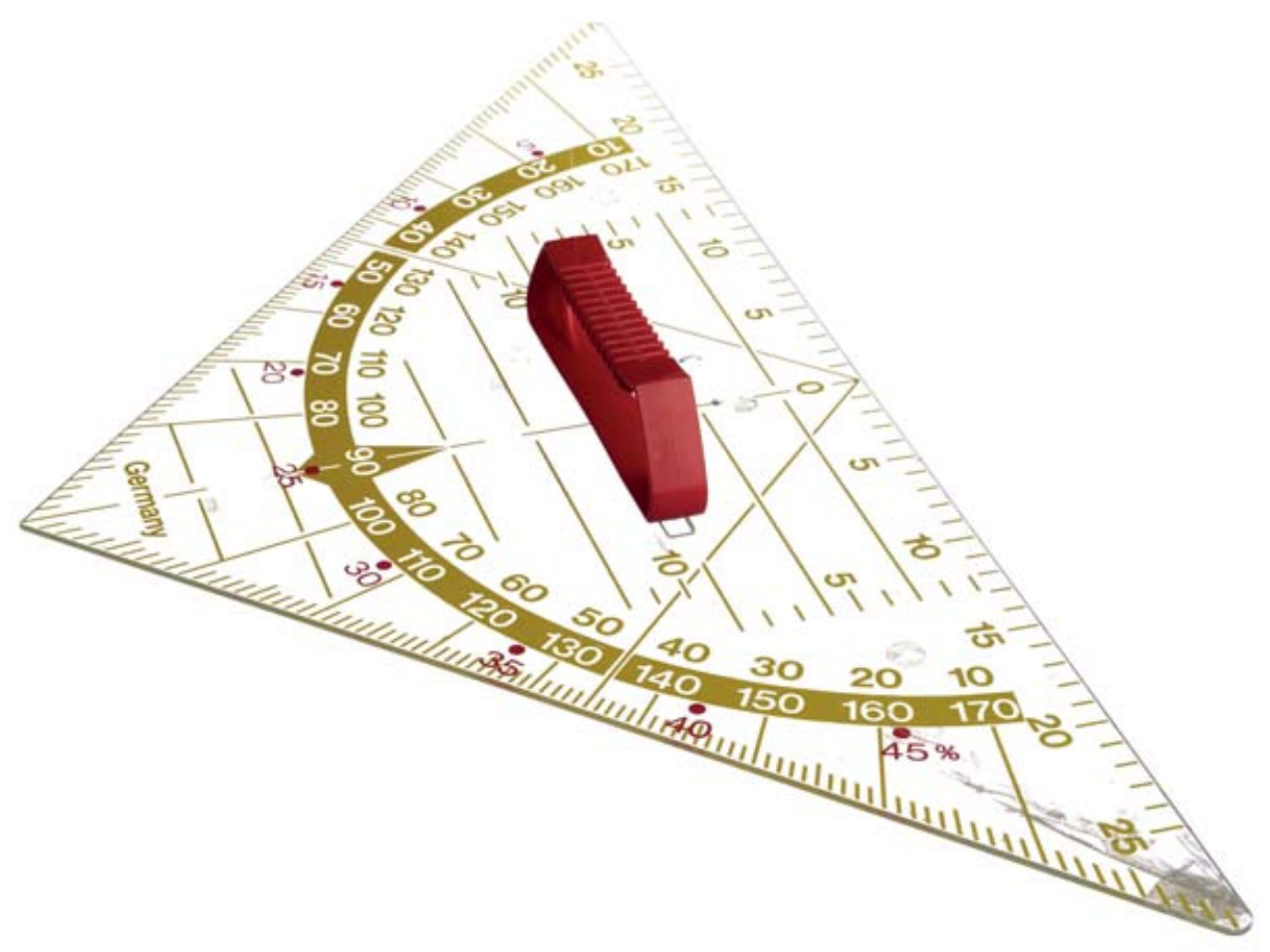

aufgrund der äußerlichen Gegebenheiten leider in diesem Fall nicht möglich.

Wie gesagt unterrichte ich an einem gut situierten Gymnasium. Was bedeutet es, dort eine Klassenarbeit wiederholen zu müssen? Genau - viel Ärger mit den Eltern, die sich einerseits beschweren, dass man ja gar nicht solche „Päckchenaufgaben“ rechnen lassen würde, wie das damals bei ihnen war, und die einem andererseits versichern, dass die Schülerinnen und Schüler für Mathematik so motiviert seien wie noch nie.

In meinem Fall hatte dies über zehn Elterngespräche zur Folge, in denen ich den Eltern erklären durfte, dass reines „Päckchenrechnen“ nicht sinnvoll ist, und dass Mathematik durchaus mehr als das ist. Eine Tatsache, die ich auch den Schülerinnen und Schülern vermitteln möchte. Man sollte an dieser Stelle vielleicht anmerken, dass es sich nicht um einen leistungsschwachen Kurs handelt, sondern vielmehr um einen Kurs mit der Note 2,o als Zeugnisdurchschnitt im Fach Mathematik.

Die hohe Anzahl der Elterngespräche hatte zur Folge, dass wieder weniger Zeit vorhanden war, um guten (!) Unterricht vorzubereiten. Zusammenfassend könnte man also sagen, dass man mit einer Zehntagewoche auf jeden Fall mehr guten Unterricht vorbereiten könnte, denn durch die organisatorischen Gegebenheiten an den Schulen heißt Lehrerin sein gleichzeitig: Verwaltung, Mediation, Gesprächsmoderation ... Um diese Herausforderungen zu meistern, ist entweder viel Erfahrung und/oder ein gut zusammenarbeitendes und strukturiertes Kollegium notwendig.

Ich möchte damit nicht sagen, dass mein Kollegium schlecht ausgestattet ist oder nicht viele Materialien oder Ähnliches hat. Wir haben eine tolle Mathewerkstatt, in die man sich theoretisch einbuchen und dort mit den Schülerinnen und Schülern experimentieren und Mathematik anwenden kann - auch in Zusammenarbeit mit anderen Fächern. Doch leider nutzt diese Materialien niemand, sodass sie mittlerweile veraltet und unvollständig sind. AuBerdem kann man die Mathewerkstatt nicht mehr buchen, da dies aufgrund der großen Raumnot an unserer Schule nicht mehr möglich ist. Leider sind zu viele Schülerinnen und Schüler an unserer Schule, sodass die eigentlich tollen didaktisch-pädagogischen Angebote nicht mehr genutzt werden können.

Doch was bedeutet es in diesem ganzen, ich nenne es hier einmal "Chaos“, Referendarin zu sein? Nun ja, ganz einfach: nicht unbedingt guten Unterricht zu machen, sondern vielmehr den Unterricht zu machen, den die Seminarleiter sehen wollen. In meinem Fall wenig Begriffseinführung durch Klassifikation und viel Begriffsdefinition durch Spezifikation. Doch leider funktioniert das so nicht, zumal wenn man bedenkt, dass dies die Forderungen des Seminarleiters in jeder Lerngruppe sind. So wissen wir doch, zumindest sollte man es wissen, dass jede Lerngruppe andere Lernvoraussetzungen mit sich bringt und daher individuell angepasste Lernarrangements benötigt. Aber wie jeder normale Mensch, möchte auch ich mit guten Noten aus dem Referendariat herausgehen. Daher findet immer zweigeteilter Unterricht statt, der normale Unterricht und derjenige, wenn der Seminarleiter mal wieder da ist. Meine Schülerinnen und Schüler wissen das mittlerweile schon und sind darauf eingestellt. Aber ist das wirklich der Sinn und Zweck einer guten Ausbildung?

Mein Fazit: Ohne mein eigenes Interesse an Mathematik und Mathematikdidaktik und meinen Willen, mir die 
Inhalte selbstständig beizubringen, würde ich nach dem Referendariat wohl doch eher auf die „Päckchenaufgaben“ zurückgreifen. Wieso? - Ganz einfach: Weil es einfacher und bequemer ist; vor allem bei nichtfunktionierender Teamarbeit im Kollegium und organisatorischem "Chaos", das man so nebenher noch bewältigen muss.

\section{Eine Mathematiklehrerin an einem Berliner Gymnasium erzählt}

Meine 8. Klasse am Gymnasium hat viel zu tun: Vorträge halten, Arbeiten schreiben, Vokabeln lernen, usw. und vielleicht noch ein zwei Hobbys, etwa Sport und Musik. Da bleibt wenig Zeit, auch noch ein Mathematiktrainingsprogramm zu durchlaufen. Seit G8 sind die Schultage auch so lang und inzwischen sind die Schülerinnen und Schüler immer jünger, was das Begreifen von Sachstrukturen und ein termingerechtes selbstständiges Arbeiten erschwert. Die Mathematikstunden sollen motivierend und an die Lebenswelt der Kinder angebunden sein, sonst sind sie ja langweilig und werden ausgeblendet. Die Welt tickt immer schneller und sensationeller. So entwickeln auch unsere Schülerinnnen und Schüler die Einstellung, unterhalten zu werden und sich nicht mehr selbst anstrengen zu wollen, um Ziele zu erreichen.

In der Oberstufe ist es nicht viel anders. Hier haben wir die Situation, dass sich die Jugendlichen breit aufstellen müssen, um für das zukünftige Leben gewappnet zu sein. Das verhindert Tiefe im Detail. Aber ist es nicht auch gewollt, den Jugendlichen eine Vielfalt mitzugeben, ehe sie sich speziell entwickeln? Was nützt es, wenn alle Schülerinnen und Schüler lernen, eine Kurvendiskussion perfekt ablaufen zu lassen, wenn sie anschließend kaum noch etwas mit Mathematik zu tun haben werden? Da ist doch Verständnis in Strukturen viel wichtiger!

Wenn Professoren seit Jahrzehnten in den Einstiegstests des Ingenieursstudiums Bruchgleichungen lösen lassen, das Thema aber schon im Rahmenlehrplan der Sekundarstufe I seit 2006 nicht mehr vorkommt und auch im aktuellen neuen Rahmenlehrplan nicht erschienen ist, muss man sich doch nicht wundern, wenn angehende Studierende diese nicht beherrschen. Daraus lässt sich nicht schlieBen, dass der Mathematikunterricht schlechter geworden ist. Wenn man Bruchgleichungen nie gelöst hat, lassen sie sich eben nicht simpel auf die Bruchrechnung zurückführen.

Die Inhalte aus dem aktuellen Rahmenlehrplan sind kaum zu schaffen, da bleibt keine Zeit für Extrathemen, was wir Lehrkräfte auch sehr bedauern. In den Bildungsstandards der KMK wird gefordert, dass die allgemeine Hochschulreife zur Studierfähigkeit führen soll. Vielleicht sollte man neu definieren, was man darunter versteht. Dabei geht es um die Fähigkeit und nicht um konkret angehäuftes Vorwissen, oder?

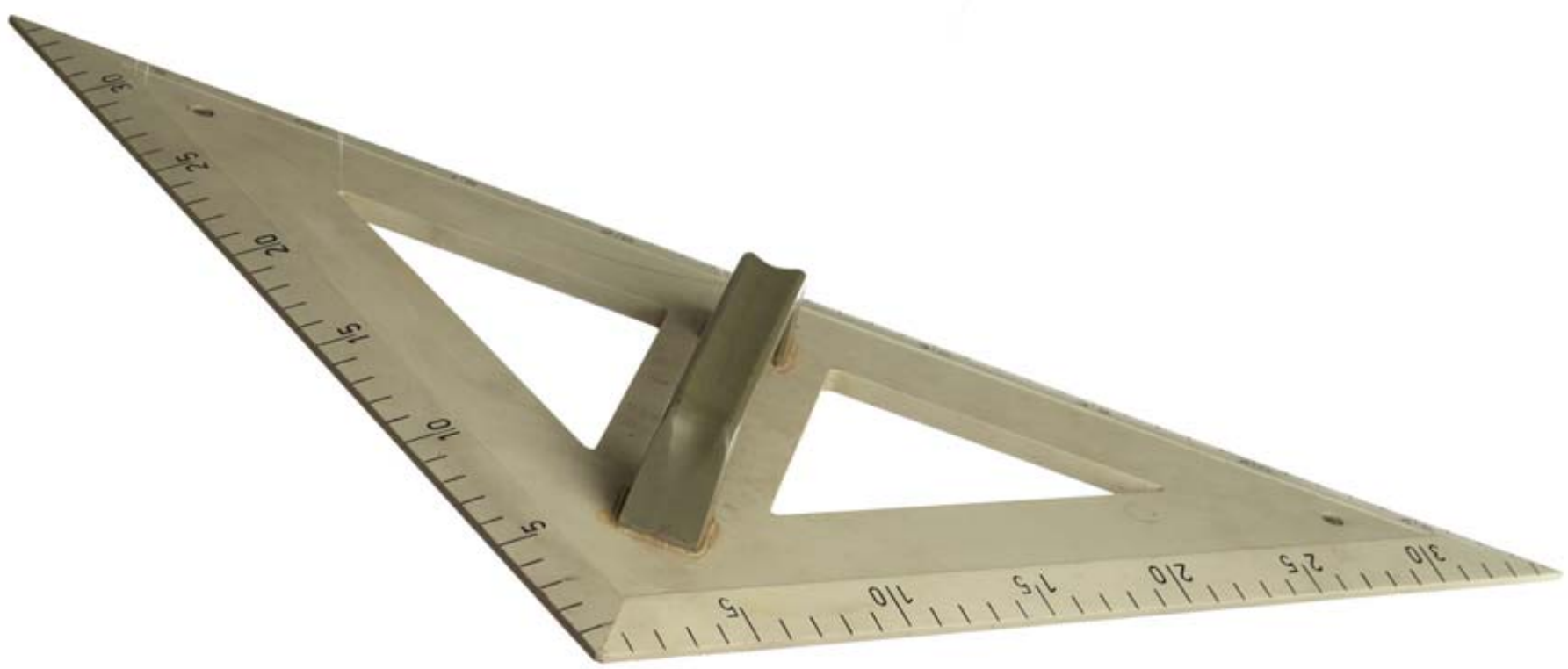

Prof. Dr. Brigitte Lutz-Westphal, Didaktik der Mathematik, Fachbereich Mathematik und Informatik, Freie Universität Berlin, Arnimallee 3, 14195 Berlin.brigitte.lutz-westphal@math.fu-berlin.de (Fotos: Christoph Eyrich) 